\title{
Hadamard type inequalities for $\varphi$-convex functions on the co-ordinates
}

\author{
Erhan Set $^{1}$, Mehmet Zeki Sarıkaya ${ }^{2}$ and Ahmet Ocak Akdemir ${ }^{3}$ \\ ${ }^{1}$ Department of Mathematics, Faculty of Science and Arts, Ordu University, Ordu, Turkey \\ ${ }^{2}$ Department of Mathematics, Faculty of Science and Arts, Düzce University, Düzce, Turkey \\ ${ }^{3}$ Department of Mathematics, Faculty of Science and Letters, A $\breve{g}$ rı İbrahim Çeçen University, Ağrı, Turkey \\ E-mail: erhanset@yahoo.com, sarikayamz@gmail.com, ahmetakdemir@agri.edu.tr
}

\begin{abstract}
In this paper, we introduce the notation of $\varphi$-convex functions on the co-ordinates and present some properties. Also, new Hadamard type inequalities for such functions are obtained.
\end{abstract}

2010 Mathematics Subject Classification. 26A51. 26D07, 26D10, 26D15

Keywords. Hadamard type inequalities, co-ordinated $\varphi$-convex functions.

\section{Introduction}

Let us recall some known definitions and results which we will use in this paper. If $f$ is a convex function on the interval $I=[a, b]$ and $a, b \in I$ with $a<b$, then

$$
f\left(\frac{a+b}{2}\right) \leq \frac{1}{b-a} \int_{a}^{b} f(x) d x \leq \frac{f(a)+f(b)}{2}
$$

which is known as the Hadamard inequality in the literature. (see, e.g., [4], [8, p.137]) If the function $f$ is concave, reversed signs of inequality hold in (1.1). The inequality (1.1) is one of the most useful inequalities in mathematical analysis. For new proofs, numerious generalizatons, variants and extensions on this inequality, see $([3]-[8])$ where further references are given.

Let us consider a function $\varphi:[a, b] \rightarrow[a, b]$ where $[a, b] \subset \mathbb{R}$. A function $f:[a, b] \rightarrow \mathbb{R}$ is said to be $\varphi$-convex on $[a, b]$ if for every two points $x, y \in[a, b]$ and $t \in[0,1]$ the following inequality holds:

$$
f(t \varphi(x)+(1-t) \varphi(y)) \leq t f(\varphi(x))+(1-t) f(\varphi(y))
$$

(see $[9])$

In [2], Cristescu established the following results for the $\varphi$-convex functions.

Lemma 1.1. For $f:(a, b) \rightarrow \mathbb{R}$, the following statements are equivalent:

(i) $f$ is $\varphi$-convex functions on $[a, b]$

(ii) for every $x, y \in[a, b]$, the mapping $g:[0,1] \rightarrow \mathbb{R}, g(t)=f(t \varphi(x)+(1-t) \varphi(y))$ is classically convex on $[0,1]$.

Obviously, if function $\varphi$ is the identity, then the classical convexity is obtained from the previous definition. For many properties of the $\varphi$-convex functions, see [1],[2],[9], [11]-[13].

In [2], Cristescu proved the following inequalities for $\varphi$-convex functions: 
Theorem 1.2. If $f:[a, b] \rightarrow \mathbb{R}$ is $\varphi$-convex for the continuous function $\varphi:[a, b] \rightarrow[a, b]$ then

$$
f\left(\frac{\varphi(a)+\varphi(b)}{2}\right) \leq \frac{1}{\varphi(b)-\varphi(a)} \int_{\varphi(a)}^{\varphi(b)} f(x) d x \leq \frac{f(\varphi(a))+f(\varphi(b))}{2} .
$$

Theorem 1.3. Let $f:[a, b] \rightarrow \mathbb{R}, g:[a, b] \rightarrow \mathbb{R}$ be real-valued, nonnegative and $\varphi$-convex functions for $\varphi:[a, b] \rightarrow[a, b]$ non-decreasing on $[a, b]$. Then

$$
\begin{gathered}
\frac{1}{\varphi(b)-\varphi(a)} \int_{\varphi(a)}^{\varphi(b)} f(x) g(x) d x \leq \frac{1}{3} M(a, b)+\frac{1}{6} N(a, b) \\
2 f\left(\frac{\varphi(a)+\varphi(b)}{2}\right) g\left(\frac{\varphi(a)+\varphi(b)}{2}\right) \\
\leq \frac{1}{\varphi(b)-\varphi(a)} \int_{\varphi(a)}^{\varphi(b)} f(x) d x+\frac{1}{6} M(a, b)+\frac{1}{3} N(a, b)
\end{gathered}
$$

where

$$
\begin{aligned}
& M(a, b)=f(\varphi(a)) g(\varphi(a))+f(\varphi(b)) g(\varphi(b)) \\
& N(a, b)=f(\varphi(a)) g(\varphi(b))+f(\varphi(b)) g(\varphi(a)) .
\end{aligned}
$$

A modification for convex functions which is also known as convex functions on $\Delta$ was introduced by Dragomir in [4] as following:

Definition 1.4. Let us now consider a bidimensional interval $\Delta=:[a, b] \times[c, d]$ in $\mathbb{R}^{2}$ with $a<b$ and $c<d$. A mapping $f: \Delta \rightarrow \mathbb{R}$ is said to be convex on $\Delta$ if the following inequality:

$$
f(\alpha x+(1-\alpha) z, \alpha y+(1-\alpha) w) \leq \alpha f(x, y)+(1-\alpha) f(z, w)
$$

holds, for all $(x, y),(z, w) \in \Delta$ and $\alpha \in[0,1]$. If the inequality reversed then $f$ is said to be concave on $\Delta$. A function $f: \Delta \rightarrow \mathbb{R}$ is said to be convex on the co-ordinates on $\Delta$ if the partial mappings $f_{y}:[a, b] \rightarrow \mathbb{R}, f_{y}(u)=f(u, y)$ and $f_{x}:[c, d] \rightarrow \mathbb{R}, f_{x}(v)=f(x, v)$ are convex where defined for all $x \in[a, b], y \in[c, d]$.

A formal definition for co-ordinated convex functions may be stated as follow [see [10]]:

Definition 1.5. A function $f: \Delta \rightarrow \mathbb{R}$ is said to be convex on the co-ordinates on $\Delta$ if the following inequality:

$$
\begin{aligned}
& f(t x+(1-t) y, s u+(1-s) w) \\
& \leq t s f(x, u)+t(1-s) f(x, w)+s(1-t) f(y, u)+(1-t)(1-s) f(y, w)
\end{aligned}
$$

holds for all $t, s \in[0,1]$ and $(x, u),(x, w),(y, u),(y, w) \in \Delta$.

The main aim of this paper is to define $\varphi$-convex functions on the co-ordinates and to give some properties of this class of functions. Some new inequalities of Hadamard type and inequalities involving product of two $\varphi$-convex functions are given. 


\section{Main Results}

Let us consider two functions $\varphi_{1}:[a, b] \rightarrow[a, b]$ and $\varphi_{2}:[c, d] \rightarrow[c, d]$ where $[a, b],[c, d] \subset \mathbb{R}$.

Definition 2.1. Let $\Delta=:[a, b] \times[c, d] \subseteq \mathbb{R}^{2}$ with $a<b$ and $c<d$. A mapping $f: \Delta \rightarrow \mathbb{R}$ is said to be $\varphi$-convex on $\Delta$ for every two points $(x, y),(z, w) \in \Delta$ and $\lambda \in[0,1]$ the following inequality holds:

$$
\begin{aligned}
& f\left(\lambda \varphi_{1}(x)+(1-\lambda) \varphi_{1}(z), \lambda \varphi_{2}(y)+(1-\lambda) \varphi_{2}(w)\right. \\
\leq \quad & \lambda f\left(\varphi_{1}(x), \varphi_{2}(y)\right)+(1-\lambda) f\left(\varphi_{1}(z), \varphi_{2}(w)\right) .
\end{aligned}
$$

A function $f: \Delta \rightarrow \mathbb{R}$ is $\varphi$-convex on $\Delta$ will be called $\varphi$-convex on co-ordinates on $\Delta$, if the partial mappings $f_{\varphi_{2}}:[a, b] \rightarrow \mathbb{R}, f_{\varphi_{2}}(u)=f\left(u, \varphi_{2}\right)$ and $f_{\varphi_{1}}:[c, d] \rightarrow \mathbb{R}, f_{\varphi_{1}}(v)=f\left(\varphi_{1}, v\right)$ are $\varphi$-convex where defined for all $\varphi_{1} \in[a, b]$ and $\varphi_{2} \in[c, d]$.

Lemma 2.2. For $f: \Delta \rightarrow \mathbb{R}$, the following statements are equivalent:

(i) $f$ is $\varphi$-convex functions on $\Delta$

(ii) for every $x, z \in[a, b], y, w \in[c, d]$ the mapping $g:[0,1] \times[0,1] \rightarrow \mathbb{R}, g\left(t_{1}, t_{2}\right)=f\left(t_{1} \varphi_{1}(x)+\right.$ $\left.\left(1-t_{1}\right) \varphi_{1}(z), t_{2} \varphi_{2}(y)+\left(1-t_{2}\right) \varphi_{2}(w)\right)$ is convex on $[0,1] \times[0,1]$.

Proof. Let us consider two points $(x, y),(z, w) \in \Delta, \lambda \in[0,1]$ and $\left(t_{1}, t_{2}\right),\left(t_{3}, t_{4}\right) \in[0,1] \times[0,1]$. Then

$$
\begin{aligned}
& g\left(\lambda t_{1}+(1-\lambda) t_{2}, \lambda t_{3}+(1-\lambda) t_{4}\right) \\
= & f\left(\left[\lambda t_{1}+(1-\lambda) t_{2}\right] \varphi_{1}(x)+\left(1-\left(\lambda t_{1}+(1-\lambda) t_{2}\right)\right) \varphi_{1}(z),\right. \\
& {\left.\left[\lambda t_{3}+(1-\lambda) t_{4}\right] \varphi_{2}(y)+\left(1-\left(\lambda t_{3}+(1-\lambda) t_{4}\right)\right) \varphi_{2}(w)\right) } \\
= & f\left(\lambda\left[t_{1} \varphi_{1}(x)+\left(1-t_{1}\right) \varphi_{1}(z)\right]+(1-\lambda)\left[t_{2} \varphi_{1}(x)+\left(1-t_{2}\right) \varphi_{1}(z)\right]\right. \\
& \left.+\lambda\left[t_{3} \varphi_{2}(y)+\left(1-t_{3}\right) \varphi_{2}(w)\right]+(1-\lambda)\left[t_{4} \varphi_{2}(y)+\left(1-t_{4}\right) \varphi_{2}(w)\right]\right) \\
\leq \quad & \lambda f\left(t_{1} \varphi_{1}(x)+\left(1-t_{1}\right) \varphi_{1}(z), t_{3} \varphi_{2}(y)+\left(1-t_{3}\right) \varphi_{2}(w)\right) \\
& +(1-\lambda) f\left(t_{2} \varphi_{1}(x)+\left(1-t_{2}\right) \varphi_{1}(z), t_{4} \varphi_{2}(y)+\left(1-t_{4}\right) \varphi_{2}(w)\right) \\
= & \lambda g\left(t_{1}, t_{3}\right)+(1-\lambda) g\left(t_{2}, t_{4}\right)
\end{aligned}
$$

meaning that $g$ is convex on $[0,1] \times[0,1]$. 
Conversely, assumimg that $g$ is convex on $[0,1] \times[0,1]$, for $x, z \in[a, b], y, w \in[c, d]$ and $\left(\varphi_{1}(x), \varphi_{2}(y)\right)=(1,1),\left(\varphi_{1}(z), \varphi_{2}(w)\right)=(0,0)$ with $\lambda \in[0,1]$ one gets

$$
\begin{aligned}
& f\left(\lambda \varphi_{1}(x)+(1-\lambda) \varphi_{1}(z), \lambda \varphi_{2}(y)+(1-\lambda) \varphi_{2}(w)\right) \\
= & g(\lambda 1+(1-\lambda) 0, \lambda 1+(1-\lambda) 0) \\
\leq & \lambda g(1,1)+(1-\lambda) g(0,0) \\
= & \lambda f\left(\varphi_{1}(x), \varphi_{2}(y)\right)+(1-\lambda) f\left(\varphi_{1}(z), \varphi_{2}(w)\right)
\end{aligned}
$$

meaning the $\varphi$-convexity of $f$.

The notion of co-ordinated $\varphi$-convexity can be given as follow:

Definition 2.3. Let $\Delta=[a, b] \times[c, d] \subseteq \mathbb{R}^{2}$ with $a<b$ and $c<d$. A mapping $f: \Delta \rightarrow \mathbb{R}$ is said to be $\varphi$-convex on the co-ordinates on $\Delta$ for all $t, s \in[0,1]$ and $(x, u),(x, v),(y, u),(y, v) \in \Delta$ if the following inequality holds:

$$
\begin{aligned}
& f\left(t \varphi_{1}(x)+(1-t) \varphi_{1}(y), s \varphi_{2}(u)+(1-s) \varphi_{2}(v)\right) \\
\leq & t s f\left(\varphi_{1}(x), \varphi_{2}(u)\right)+t(1-s) f\left(\varphi_{1}(x), \varphi_{2}(v)\right) \\
\quad & s(1-t) f\left(\varphi_{1}(y), \varphi_{2}(u)\right)+(1-t)(1-s) f\left(\varphi_{1}(y), \varphi_{2}(v)\right) .
\end{aligned}
$$

Theorem 2.4. Suppose that $f: \Delta=[a, b] \times[c, d] \subseteq \mathbb{R}^{2} \rightarrow \mathbb{R}$ is $\varphi$-convex on the co-ordinates on $\Delta$ with $f \in L[\Delta]$, then one has the inequalities:

$$
\begin{aligned}
& f\left(\frac{\varphi_{1}(a)+\varphi_{1}(b)}{2}, \frac{\varphi_{2}(c)+\varphi_{2}(d)}{2}\right) \\
\leq & \frac{1}{\left(\varphi_{1}(b)-\varphi_{1}(a)\right)\left(\varphi_{2}(d)-\varphi_{2}(c)\right)} \int_{\varphi_{1}(a)}^{\varphi_{1}(b)} \int_{\varphi_{2}(c)}^{\varphi_{2}(d)} f(x, y) d y d x \\
\leq & \frac{f\left(\varphi_{1}(a), \varphi_{2}(c)\right)+f\left(\varphi_{1}(a), \varphi_{2}(d)\right)+f\left(\varphi_{1}(b), \varphi_{2}(c)\right)+f\left(\varphi_{1}(b), \varphi_{2}(d)\right)}{4} .
\end{aligned}
$$

Proof. In order to prove of the first part of (2.2), we start with the following calculus, $(t, s) \in$ 
$[0,1] \times[0,1]$ using the $\varphi$-convexity on the co-ordinates on $\Delta$ of the given function

$$
\begin{aligned}
& f\left(\frac{\varphi_{1}(a)+\varphi_{1}(b)}{2}, \frac{\varphi_{2}(c)+\varphi_{2}(d)}{2}\right) \\
= & f\left(\frac{t \varphi_{1}(a)+(1-t) \varphi_{1}(b)}{2}+\frac{t \varphi_{1}(b)+(1-t) \varphi_{1}(a)}{2},\right. \\
& \left.\frac{s \varphi_{2}(c)+(1-s) \varphi_{2}(d)}{2}+\frac{s \varphi_{2}(d)+(1-s) \varphi_{2}(c)}{2}\right) \\
\leq \quad & \frac{1}{4}\left[f\left(t \varphi_{1}(a)+(1-t) \varphi_{1}(b), s \varphi_{2}(c)+(1-s) \varphi_{2}(d)\right)\right. \\
& +f\left(t \varphi_{1}(a)+(1-t) \varphi_{1}(b), s \varphi_{2}(d)+(1-s) \varphi_{2}(c)\right) \\
& +f\left(t \varphi_{1}(b)+(1-t) \varphi_{1}(a), s \varphi_{2}(c)+(1-s) \varphi_{2}(d)\right) \\
& \left.+f\left(t \varphi_{1}(b)+\left(1-t_{1}\right) \varphi_{1}(a), s \varphi_{2}(d)+(1-s) \varphi_{2}(c)\right)\right]
\end{aligned}
$$

Lemma 2.2 allows us to integrate both sides of this inequality over $[0,1] \times[0,1]$ which lead to,

$$
\begin{aligned}
& f\left(\frac{\varphi_{1}(a)+\varphi_{1}(b)}{2}, \frac{\varphi_{2}(c)+\varphi_{2}(d)}{2}\right) \\
\leq & \frac{1}{4}\left[\int_{0}^{1} \int_{0}^{1} f\left(t \varphi_{1}(a)+(1-t) \varphi_{1}(b), s \varphi_{2}(c)+(1-s) \varphi_{2}(d)\right) d s d t\right. \\
+ & \int_{0}^{1} \int_{0}^{1} f\left(t \varphi_{1}(a)+(1-t) \varphi_{1}(b), s \varphi_{2}(d)+(1-s) \varphi_{2}(c)\right) d s d t \\
+ & \int_{0}^{1} \int_{0}^{1} f\left(t \varphi_{1}(b)+(1-t) \varphi_{1}(a), s \varphi_{2}(c)+(1-s) \varphi_{2}(d)\right) d s d t \\
& \left.+\int_{0}^{1} \int_{0}^{1} f\left(t \varphi_{1}(b)+(1-t) \varphi_{1}(a), s \varphi_{2}(d)+(1-s) \varphi_{2}(c)\right)\right] d s d t .
\end{aligned}
$$

By changing of the variables in the above inequality, we obtain

$$
\begin{aligned}
& f\left(\frac{\varphi_{1}(a)+\varphi_{1}(b)}{2}, \frac{\varphi_{2}(c)+\varphi_{2}(d)}{2}\right) \\
\leq & \frac{1}{\left(\varphi_{1}(b)-\varphi_{1}(a)\right)\left(\varphi_{2}(d)-\varphi_{2}(c)\right)} \int_{\varphi_{1}(a)}^{\varphi_{1}(b)} \int_{\varphi_{2}(c)}^{\varphi_{2}(d)} f(x, y) d y d x,
\end{aligned}
$$


which is the first inequality of the inequality (2.2).

For the proof of the second inequality of the inequality (2.2), we use the (2.1) with setting $x=a$, $y=b, u=c$ and $v=d$, we have

$$
\begin{aligned}
& f\left(t \varphi_{1}(a)+(1-t) \varphi_{1}(b), s \varphi_{2}(c)+(1-s) \varphi_{2}(d)\right) \\
\leq & t s f\left(\varphi_{1}(a), \varphi_{2}(c)\right)+t(1-s) f\left(\varphi_{1}(a), \varphi_{2}(d)\right) \\
& +s(1-t) f\left(\varphi_{1}(b), \varphi_{2}(c)\right)+(1-t)(1-s) f\left(\varphi_{1}(b), \varphi_{2}(d)\right) .
\end{aligned}
$$

Lemma 2.2 implies the integrability with respect to $(t, s)$ over $[0,1] \times[0,1]$ of both sides of the previous inequality,

$$
\begin{aligned}
& \int_{0}^{1} \int_{0}^{1} f\left(t \varphi_{1}(a)+(1-t) \varphi_{1}(b), s \varphi_{2}(c)+(1-s) \varphi_{2}(d)\right) d s d t \\
\leq & \int_{0}^{1} \int_{0}^{1} t s f\left(\varphi_{1}(a), \varphi_{2}(c)\right) d s d t+\int_{0}^{1} \int_{0}^{1} t(1-s) f\left(\varphi_{1}(a), \varphi_{2}(d)\right) d s d t \\
& +\int_{0}^{1} \int_{0}^{1} s(1-t) f\left(\varphi_{1}(b), \varphi_{2}(c)\right) d s d t+\int_{0}^{1} \int_{0}^{1}(1-t)(1-s) f\left(\varphi_{1}(b), \varphi_{2}(d)\right) d s d t .
\end{aligned}
$$

By a simple computation and by using the change of the variables, we obtain the required result.

Theorem 2.5. Let $f, g: \Delta=[a, b] \times[c, d] \subseteq \mathbb{R}^{2} \rightarrow[0, \infty)^{2}$ are $\varphi$-convex functions on the coordinates on $\Delta$ with $f g \in L[\Delta]$, then one has the inequality:

$$
\begin{aligned}
& \frac{1}{\left(\varphi_{1}(b)-\varphi_{1}(a)\right)\left(\varphi_{2}(d)-\varphi_{2}(c)\right)} \int_{\varphi_{1}(a)}^{\varphi_{1}(b)} \int_{\varphi_{2}(c)}^{\varphi_{2}(d)} f(x, y) g(x, y) d y d x \\
\leq & \frac{1}{9} L(a, b, c, d)+\frac{1}{36} N(a, b, c, d)+\frac{1}{18} M(a, b, c, d)
\end{aligned}
$$

where

$$
\begin{aligned}
& L(a, b, c, d) \\
=\quad & f\left(\varphi_{1}(a), \varphi_{2}(c)\right) g\left(\varphi_{1}(a), \varphi_{2}(c)\right)+f\left(\varphi_{1}(b), \varphi_{2}(c)\right) g\left(\varphi_{1}(b), \varphi_{2}(c)\right) \\
& +f\left(\varphi_{1}(a), \varphi_{2}(d)\right) g\left(\varphi_{1}(a), \varphi_{2}(d)\right)+f\left(\varphi_{1}(b), \varphi_{2}(d)\right) g\left(\varphi_{1}(b), \varphi_{2}(d)\right)
\end{aligned}
$$




$$
\begin{aligned}
& M(a, b, c, d) \\
= & f\left(\varphi_{1}(a), \varphi_{2}(c)\right) g\left(\varphi_{1}(a), \varphi_{2}(d)\right)+f\left(\varphi_{1}(a), \varphi_{2}(d)\right) g\left(\varphi_{1}(a), \varphi_{2}(c)\right) \\
& +f\left(\varphi_{1}(b), \varphi_{2}(c)\right) g\left(\varphi_{1}(b), \varphi_{2}(d)\right)+f\left(\varphi_{1}(b), \varphi_{2}(d)\right) g\left(\varphi_{1}(b), \varphi_{2}(c)\right) \\
& +f\left(\varphi_{1}(b), \varphi_{2}(c)\right) g\left(\varphi_{1}(a), \varphi_{2}(c)\right)+f\left(\varphi_{1}(b), \varphi_{2}(d)\right) g\left(\varphi_{1}(a), \varphi_{2}(d)\right) \\
& +f\left(\varphi_{1}(a), \varphi_{2}(c)\right) g\left(\varphi_{1}(b), \varphi_{2}(c)\right)+f\left(\varphi_{1}(a), \varphi_{2}(d)\right) g\left(\varphi_{1}(b), \varphi_{2}(d)\right)
\end{aligned}
$$

and

$$
\begin{aligned}
& N(a, b, c, d) \\
=\quad & f\left(\varphi_{1}(b), \varphi_{2}(c)\right) g\left(\varphi_{1}(a), \varphi_{2}(d)\right)+f\left(\varphi_{1}(b), \varphi_{2}(d)\right) g\left(\varphi_{1}(a), \varphi_{2}(c)\right) \\
& +f\left(\varphi_{1}(a), \varphi_{2}(c)\right) g\left(\varphi_{1}(b), \varphi_{2}(d)\right)+f\left(\varphi_{1}(a), \varphi_{2}(d)\right) g\left(\varphi_{1}(b), \varphi_{2}(c)\right) .
\end{aligned}
$$

Proof. Since $f, g$ are $\varphi$-convex on the co-ordinates on $\Delta$, we have

$$
\begin{aligned}
& f\left(t \varphi_{1}(a)+(1-t) \varphi_{1}(b), s \varphi_{2}(c)+(1-s) \varphi_{2}(d)\right) \\
\leq \quad & t s f\left(\varphi_{1}(a), \varphi_{2}(c)\right)+t(1-s) f\left(\varphi_{1}(a), \varphi_{2}(d)\right) \\
& +s(1-t) f\left(\varphi_{1}(b), \varphi_{2}(c)\right)+(1-t)(1-s) f\left(\varphi_{1}(b), \varphi_{2}(d)\right)
\end{aligned}
$$

and

$$
\begin{aligned}
& g\left(t \varphi_{1}(a)+(1-t) \varphi_{1}(b), s \varphi_{2}(c)+(1-s) \varphi_{2}(d)\right) \\
\leq \quad & t s g\left(\varphi_{1}(a), \varphi_{2}(c)\right)+t(1-s) g\left(\varphi_{1}(a), \varphi_{2}(d)\right) \\
\quad & +s(1-t) g\left(\varphi_{1}(b), \varphi_{2}(c)\right)+(1-t)(1-s) g\left(\varphi_{1}(b), \varphi_{2}(d)\right)
\end{aligned}
$$

for all $(t, s) \in[0,1] \times[0,1]$. Since $f$ and $g$ are non-negative, if we multiply the above inequalities 
side by side, we get

$$
\begin{aligned}
& f\left(t \varphi_{1}(a)+(1-t) \varphi_{1}(b), s \varphi_{2}(c)+(1-s) \varphi_{2}(d)\right) \\
& \times g\left(t \varphi_{1}(a)+(1-t) \varphi_{1}(b), s \varphi_{2}(c)+(1-s) \varphi_{2}(d)\right) \\
& \leq\left[t s f\left(\varphi_{1}(a), \varphi_{2}(c)\right)+t(1-s) f\left(\varphi_{1}(a), \varphi_{2}(d)\right)\right. \\
& \left.+s(1-t) f\left(\varphi_{1}(b), \varphi_{2}(c)\right)+(1-t)(1-s) f\left(\varphi_{1}(b), \varphi_{2}(d)\right)\right] \\
& \times\left[t s g\left(\varphi_{1}(a), \varphi_{2}(c)\right)+t(1-s) g\left(\varphi_{1}(a), \varphi_{2}(d)\right)\right. \\
& \left.+s(1-t) g\left(\varphi_{1}(b), \varphi_{2}(c)\right)+(1-t)(1-s) g\left(\varphi_{1}(b), \varphi_{2}(d)\right)\right] .
\end{aligned}
$$

Then, if we integrate the both side of the above inequality with respect to $(t, s)$ over $[0,1] \times[0,1]$, we have the inequality in (2.3).

Theorem 2.6. Let $f, g: \Delta=[a, b] \times[c, d] \subset \mathbb{R}^{2} \rightarrow \mathbb{R}$ be $\varphi$-convex functions on $\Delta$ with $a<b$, $c<d$ and $f g \in L[\Delta]$. Then one has the inequality:

$$
\begin{aligned}
& 2 f\left(\frac{\varphi_{1}(a)+\varphi_{1}(b)}{2}, \frac{\varphi_{2}(c)+\varphi_{2}(d)}{2}\right) g\left(\frac{\varphi_{1}(a)+\varphi_{1}(b)}{2}, \frac{\varphi_{2}(c)+\varphi_{2}(d)}{2}\right) \\
\leq & \frac{1}{\left(\varphi_{1}(b)-\varphi_{1}(a)\right)\left(\varphi_{2}(d)-\varphi_{2}(c)\right)} \int_{\varphi_{1}(a)}^{\varphi_{1}(b)} \int_{\varphi_{2}(c)}^{\varphi_{2}(d)} f(x, y) g(x, y) d y d x \\
& +\frac{1}{36} L(a, b, c, d)+\frac{1}{9} N(a, b, c, d)+\frac{1}{18} M(a, b, c, d)
\end{aligned}
$$

where $L(a, b, c, d), M(a, b, c, d), N(a, b, c, d)$ by defined as in Theorem 2.5. 
Proof. Since $f$ and $g$ are $\varphi$-convex on $\Delta$, then we observe that

$$
\begin{aligned}
& f\left(\frac{\varphi_{1}(a)+\varphi_{1}(b)}{2}, \frac{\varphi_{2}(c)+\varphi_{2}(d)}{2}\right) g\left(\frac{\varphi_{1}(a)+\varphi_{1}(b)}{2}, \frac{\varphi_{2}(c)+\varphi_{2}(d)}{2}\right) \\
& =f\left(\frac{t \varphi_{1}(a)+(1-t) \varphi_{1}(b)}{2}+\frac{t \varphi_{1}(b)+(1-t) \varphi_{1}(a)}{2},\right. \\
& \left.\frac{s \varphi_{2}(c)+(1-s) \varphi_{2}(d)}{2}+\frac{s \varphi_{2}(d)+(1-s) \varphi_{2}(c)}{2}\right) \\
& \times g\left(\frac{t \varphi_{1}(a)+(1-t) \varphi_{1}(b)}{2}+\frac{t \varphi_{1}(b)+(1-t) \varphi_{1}(a)}{2},\right. \\
& \left.\frac{s \varphi_{2}(c)+(1-s) \varphi_{2}(d)}{2}+\frac{s \varphi_{2}(d)+(1-s) \varphi_{2}(c)}{2}\right) \\
& \leq \frac{1}{4}\left[f\left(t \varphi_{1}(a)+(1-t) \varphi_{1}(b), s \varphi_{2}(c)+(1-s) \varphi_{2}(d)\right)\right. \\
& \left.+f\left(t \varphi_{1}(b)+(1-t) \varphi_{1}(a), s \varphi_{2}(d)+(1-s) \varphi_{2}(c)\right)\right] \\
& \times\left[g\left(t \varphi_{1}(a)+(1-t) \varphi_{1}(b), s \varphi_{2}(c)+(1-s) \varphi_{2}(d)\right)\right. \\
& \left.+g\left(t \varphi_{1}(b)+(1-t) \varphi_{1}(a), s \varphi_{2}(d)+(1-s) \varphi_{2}(c)\right)\right] \\
& \leq \frac{1}{4}\left[f\left(t \varphi_{1}(a)+(1-t) \varphi_{1}(b), s \varphi_{2}(c)+(1-s) \varphi_{2}(d)\right)\right. \\
& \left.\times g\left(t \varphi_{1}(a)+(1-t) \varphi_{1}(b), s \varphi_{2}(c)+(1-s) \varphi_{2}(d)\right)\right] \\
& +\frac{1}{4}\left[f\left(t \varphi_{1}(b)+(1-t) \varphi_{1}(a), s \varphi_{2}(d)+(1-s) \varphi_{2}(c)\right)\right. \\
& \left.\times g\left(t \varphi_{1}(b)+(1-t) \varphi_{1}(a), s \varphi_{2}(d)+(1-s) \varphi_{2}(c)\right)\right] \\
& +\frac{1}{4}\left[t s f\left(\varphi_{1}(a), \varphi_{2}(c)\right)+t(1-s) f\left(\varphi_{1}(a), \varphi_{2}(d)\right)\right. \\
& \left.+s(1-t) f\left(\varphi_{1}(b), \varphi_{2}(c)\right)+(1-t)(1-s) f\left(\varphi_{1}(b), \varphi_{2}(d)\right)\right] \\
& \times\left[\operatorname{tsg}\left(\varphi_{1}(b), \varphi_{2}(d)\right)+t(1-s) g\left(\varphi_{1}(b), \varphi_{2}(c)\right)\right. \\
& \left.+s(1-t) g\left(\varphi_{1}(a), \varphi_{2}(d)\right)+(1-t)(1-s) g\left(\varphi_{1}(a), \varphi_{2}(c)\right)\right] \\
& +\frac{1}{4}\left[\operatorname{tsf}\left(\varphi_{1}(b), \varphi_{2}(d)\right)+t(1-s) f\left(\varphi_{1}(b), \varphi_{2}(c)\right)\right. \\
& \left.+s(1-t) f\left(\varphi_{1}(a), \varphi_{2}(d)\right)+(1-t)(1-s) f\left(\varphi_{1}(a), \varphi_{2}(c)\right)\right] \\
& \times\left[\operatorname{tsg}\left(\varphi_{1}(a), \varphi_{2}(c)\right)+t(1-s) g\left(\varphi_{1}(a), \varphi_{2}(d)\right)\right. \\
& \left.+s(1-t) g\left(\varphi_{1}(b), \varphi_{2}(c)\right)+(1-t)(1-s) g\left(\varphi_{1}(b), \varphi_{2}(d)\right)\right] \text {. }
\end{aligned}
$$

By integrating both sides with respect to $t, s$ on $[0,1] \times[0,1]$, we get desired result.

\section{References}

[1] G. Cristescu and L. Lupşa, Non-connected convexities and applications, Kluwer Academic Publishers Dordrecht / Boston / London, 2002. 
[2] G. Cristescu, Hadamard type inequalities for $\varphi$-convex functions, Annals of the University of Oradea, Fascicle of Management and Technological Engineering, C-Rom Edition, III (XIII), 2004 .

[3] M.K. Bakula and J. Pečarić, Note on some Hadamard-type inequalities, Journal of Inequalities in Pure and Applied Mathematics, vol. 5, no. 3, article 74, 2004.

[4] S.S. Dragomir and C.E.M. Pearce, Selected Topics on Hermite-Hadamard Inequalities and Appications, RGMIA Monographs, Victoria University, 2000.

[5] S.S. Dragomir and R.P. Agarwal, Two inequalities for differentiable mappings and applications to special means of real numbers and to trapezoidal formula, Appl. Math. Lett. 11(5) (1998), 91-95.

[6] S.S. Dragomir and B. Bond, Integral inequalities of Hadamard type for log - convex functions, Demonstratio Math., 31(1998), 354-364.

[7] C.E.M. Pearce, J. Pečarić, V. Šimic, Stolarsky means and Hadamard's inequality, J. Math. Anal. Appl., 220 (1998), 99-109.

[8] J.E. Pečarić, F. Proschan, Y.L. Tong, Convex Functions, Partial Orderings, and Statistical Applications, Academic Press, INC, 1992.

[9] E.A. Youness, E-convex Sets, E-convex Functions and E-convex Programming, Journal of Optimization Theory and Applications, 102, 2(1999), 439-450.

[10] M.A. Latif and M. Alomari, On Hadamard-type inequalities for $h$-convex functions on the co-ordinates, Int. Journal of Math. Analysis, 33, 2009, 1645-1656.

[11] M. Z. Sarikaya, On Hermite Hadamard-type inequalities for strongly $\varphi$-convex functions, Southeast Asian Bull. Math., in press.

[12] M. Z. Sarikaya, On Hermite Hadamard-type inequalities for $\varphi_{h}$-convex functions, Kochi Journal of Mathematics, 9 (2014), 83-90 .

[13] M. Z. Sarikaya, On strongly $\varphi_{h}$-convex functions in inner product spaces, Arabian Journal of Mathematics, (2013) 2:295-302. 